\title{
THEORETICAL INVESTIGATION OF LIQUID METAL MHD FREE SURFACE FLOWS FOR ALPS
}

\author{
S. Molokov, I. Cox \\ Coventry University
}

C. B. Reed

Argonne National Laboratory

Fusion Power Program

\section{$14^{\text {th }}$ ANS Topical Meeting on the Technology of Fusion Energy}

October 15-19, 2000

\author{
Park City, Utah
}

The submitted manuscript has been created The submited manersity of Chicago as Operator of by the University of Chicago as Operator of Argonne National Laborat-109-ENG-38 with under Contract No. W-31-109-ENG-38 W.S. the U.S. Department of Energy. The U.S. Government retains for itself, and others acting on its behalf, a paid-up, nonexclusive, irrevocable worldwide license in said article to reproduce, prepare derivative works, disto reproduce, prepare derivalic, and perform pubtribute copies to the public, and on behalf of the Government. 


\section{DISCLAIMER}

This report was prepared as an account of work sponsored by an agency of the United States Government. Neither the United States Government nor any agency thereof, nor any of their employees, make any warranty, express or implied, or assumes any legal liability or responsibility for the accuracy, completeness, or usefulness of any information, apparatus, product, or process disclosed, or represents that its use would not infringe privately owned rights. Reference herein to any specific commercial product, process, or service by trade name, trademark, manufacturer, or otherwise does not necessarily constitute or imply its endorsement, recommendation, or favoring by the United States Government or any agency thereof. The views and opinions of authors expressed herein do not necessarily state or reflect those of the United States Government or any agency thereof. 


\section{DISCLAIMER}

Portions of this document may be illegible in electronic image products. Images are produced from the best available original document. 


\title{
THEORETICAL INVESTIGATION OF LIQUID METAL MHD FREE SURFACE FLOWS FOR ALPS
}

\author{
LOG No. PT2C06
}

RECEIVED

DEC $0 \& 2000$

\author{
S. MOLOKOV $^{1}$, I. COX ${ }^{1}$ and C.B. REED ${ }^{2}$ \\ ${ }^{1}$ Coventry University, MIS, Priory Street, Coventry CV1 5FB, UK \\ ${ }^{2}$ Argonne National Laboratory, Fusion Power Program, 9700 South Cass \\ Avenue, Argonne, IL 60439, USA
}

\begin{abstract}
Free surface plasma facing components (PFCs) offer the potential to solve the lifetime issues limiting current solid surface designs for tokamak fusion reactors by eliminating the problems of erosion and thermal stresses accompanying solid surface designs. The moving PFC free surfaces provide the possibility of absorbing impurities and possibly helium for removal outside of the plasma chamber. Free surface PFCs may also offer more creative possibilities for heat removal and higher thermal conversion efficiencies for the entire system. Design requirements for PFCs include handling $\sim 50 \%$ of the plasma heat flux and $\sim 90 \%$ of the ion flux.
\end{abstract}

Magnetohydrodynamic (MHD) liquid metal flows with free surfaces are discussed with reference to Advanced Limiter-divertor Plasma-facing Systems (ALPS) program. Specific MHD issues for the jet divertor are outlined. Results for the rivulet flow and for the thermocapillary flow in a jet are presented.

\section{Introduction}

In a tokamak reactor, liquid metals flow in a strong magnetic field $\sim 5-10 \mathrm{~T}$, which leads to a very strong MHD interaction. Liquid metal free surface PFCs can be broken down into three main groups: 1) a film flowing over a solid substrate, 2) a droplet curtain, and 3) a jet curtain. Of these three, the droplet curtain and the jet curtain are thought to be least affected by the presence of the magnetic field confining the plasma. Both droplet and jet magnetohydrodynamic (MHD) flows are definitely influenced by the presence of magnetic fields, however. Qualitatively, a uniform magnetic field tends to stabilize the geometry of a droplet or jet flow; surface instabilities are suppressed and the flow field appears to be much more "organized". A review of existing MHD-work on freesurface flows has been given in [1].
Because of the significant influence of a magnetic field on the performance of all three of these PFC concepts, it is necessary to consider MHD effects when modelling, designing and testing these free surface systems.

The advantages and disadvantages of film-, dropand jet- divertors have been discussed in [1], where it has been recommended to pursue the development of a jet divertor concept for ALPS. In this paper we summarise the most important problems to be investigated for a jet divertor, and then present theoretical results for two of them, the rivulet flow and the thermocapillary flow.

\section{Magnetohydrodynamic issues for a jet divertor}

The most important part of the jet divertor involves a liquid metal jet, which appears from a nozzle (Fig. 1a). The jet absorbs high heat flux from plasma, and falls either into a collector (Fig. 1b) or into a chute (Fig. 1c). There are several MHD issues for the jet divertor that need to be investigated, which are indicated in Fig. la-c. They are:

1. Pressure drop in the supplying duct due to a nonuniform field and bending of the duct

2. Transition from duct flow to jet flow (the nozzle problem) and the meniscus effect

3. Jet stability

4. Heat transfer analysis, including thermocapillary convection

5. Impact of a jet on a liquid metal surface

6. Impact of a jet on a solid wall

7. The problem of draining (rivulet)

Two problems are discussed below. The first one, the flow in a rivulet, is relevant to the problem of draining 7 . The second one, thermocapillary convection in a circular jet, is relevant to problem 4 . Here we 


\section{S.MOLOKOV, I.COX and C.B.REED}

discuss fundamental properties of the flows; divertor design aspects will be discussed elsewhere.
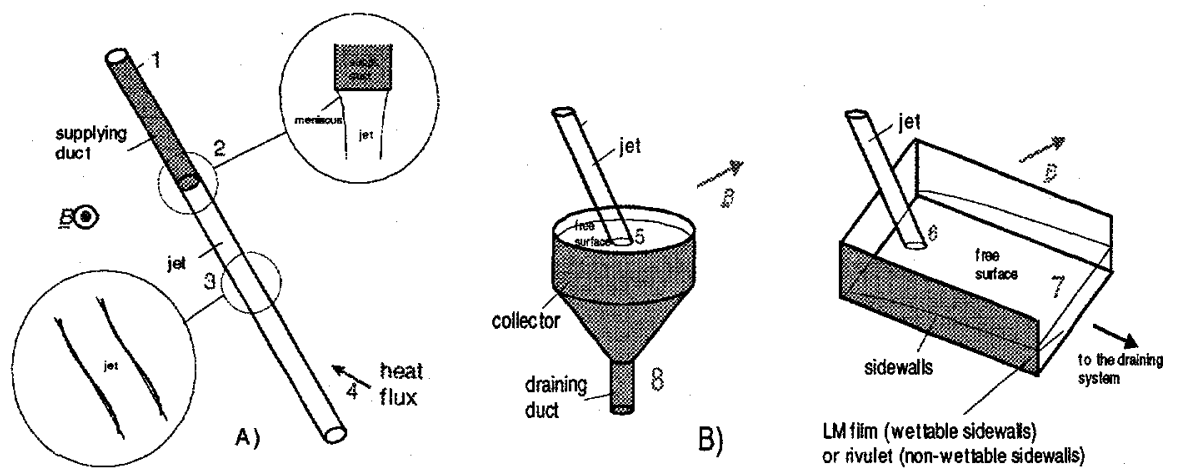

C)

Figure 1. Schematic diagram of the jet divertor: upper part (A) and two possible lower parts $(B),(C)$ of the divertor.

inclined at an angle $\alpha$ to the horizon. A strong, uniform,

\section{Flow in a rivulet}

Rivulets on a solid plate (Fig. 2) may form if wettability of either the plate or the sidewalls is poor, or as a result of the rupture of a thin liquid-metal film, as has been experimentally observed in [2], [3]. We consider fully developed MHD flow in a rivulet on an inclined plate. The plate is partially wetted, and is electrically insulating. The external magnetic field is transverse to the flow direction, and is either transverse or parallel to the plate. An asymptotic solution of the problem for high values of the Hartmann number has been obtained [4].

\subsection{FORMULATION AND SOLUTION OF THE PROBLEM}

Consider the steady, gravity-driven, isothermal, fully developed flow of a viscous, electrically conducting, incompressible fluid along the $x_{*}$-axis in a rivulet (Fig. 2). Asterisks denote dimensional quantities. A solid plate is located at $z_{*}=0$; it is

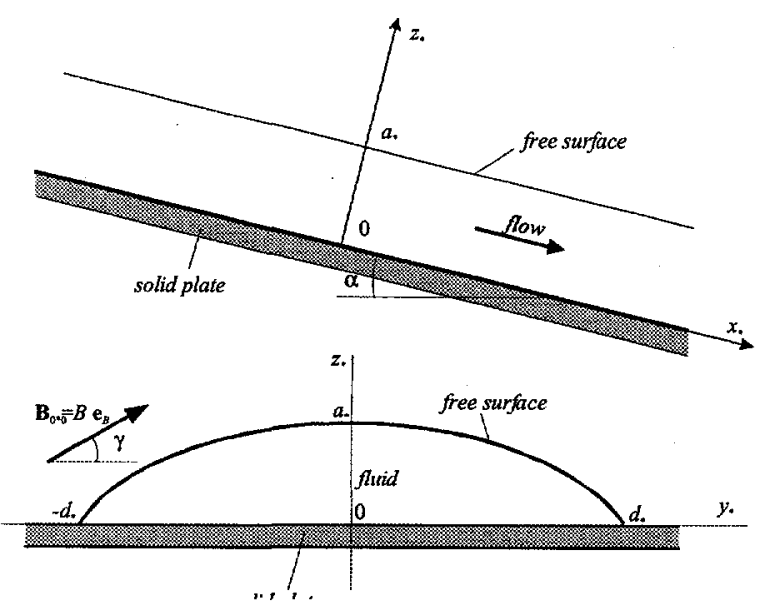
transverse magnetic field $\mathbf{B}_{0 *}=B_{0} \mathbf{e}_{B}$ is applied in the plane $\left(y_{*}, z_{*}\right)$ at an angle $\gamma$ to the horizon. The characteristic values of the length, the fluid velocity, the induced magnetic field, the electric current density, the electric potential, and the pressure are $a_{*}$, the maximum height, $v_{0}=\rho g \sin \alpha /\left(\sigma B_{0}^{\sim}\right), \sigma \mu a_{*} v_{0} B_{0}, \sigma v_{0} B_{0}, a_{*} v_{0} B_{0}$, and $a_{*} \rho g \cos \alpha$, respectively. In the above, $\sigma, \rho$, vare the electrical conductivity, density and kinematic viscosity of the fluid; $\mu$ is the magnetic permeability; $g$ is the intensity of gravity.

The solution to the problem is constructed in the following way. First, the location of the free surface, which is determined by gravity and surface tension only, is obtained. Next, for a given shape of the rivulet crosssection, the fluid velocity, the electric current density, the induced magnetic field, the electric potential, and finally the pressure are obtained using matched asymptotic expansions at high values of the Hartmann number $H a=a_{*} B_{0} \sqrt{ } \sigma / \rho v$.

\subsection{MAGNETIC FIELD TRANSVERSE TO THE PLATE}

When the fluid flows down an inclined plane in a transverse magnetic field, an $O(1)$ electric current is induced initially in the negative $y$-direction. This component of current interacts with the magnetic field to produce the Lorentz force, which balances gravity in the core. The core electric current must vanish at the free surface, and as a result, the current lines repeat the shape of the free surface (Fig. 3). The electric current completes its path in the Hartmann layer at the plate, where it is $O(H a)$ owing to current conservation. While passing through the Hartmann layer, the current induces an $O(H a)$ electric potential difference in both the layer, and the core, which must be balanced by the 
electromotive force $(u \mathbf{x}) \times \mathbf{e}_{B}$. This leads to the $O(H a)$ velocity in the core. To the leading order, $O(H a)$, the velocity profile repeats the shape of the rivulet's cross-section, similar to the flow in insulating ducts. The $O(1)$-correction to the velocity profile leads to a slight linear variation with $z$ in the core. There is no Hartmann layer at the free surface to the leading order. The Hartmann layer appears in the next, $O(1)$ approximation. The reason for its appearance is that the $O(H a)$-velocity is a function of $y$ only, and this leads to a non-zero, $O(1)$ traction at the free surface. This traction is compensated by the exponential variation of the velocity in the Hartmann layer. In dimensional terms both the local and average fluid velocities are proportional to $B_{0}{ }^{\prime}$.

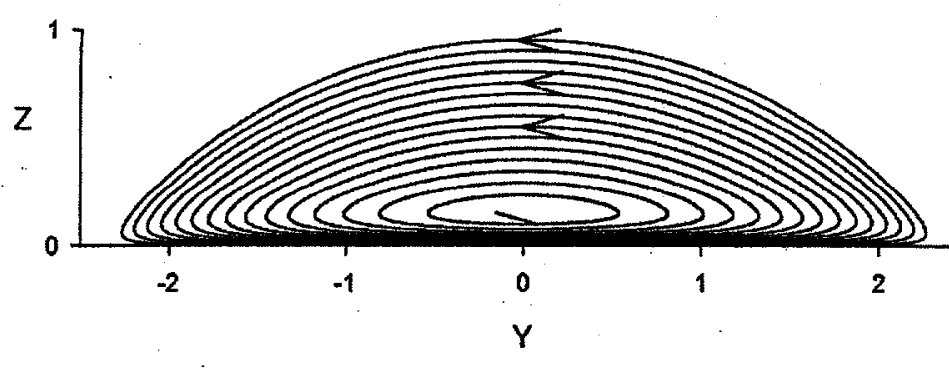

Figure 3 Rivulet in a transverse field: electric current lines for $H a=20$ and contact angle $\theta=\pi / 4$.

\subsection{MAGNETIC FIELD PARALLEL TO THE PLATE}

Since the magnetic field lines do not cross the solid boundary, the velocity in the core turns out to be $O\left(H a^{2}\right)$, i.e. independent of the magnetic field strength for sufficiently high field, and far higher than for a transverse field. The electric current is $O(1)$, and therefore, the viscous force is of the same order as the electromagnetic one. The electric current lines are shown in Fig. 4. The role of the magnetic field is to "straighten" the velocity profiles in the horizontal direction. The latter may be described by a single curve for all values of $y$. The zero-traction conditions at the free surface are satisfied in the lower-order Hartmann layers. It is important to note that in contrast to the transverse field, the flow in the bulk of the crosssection is viscous. Therefore, to the leading order the "core" occupies the whole cross-section

For both field directions, transverse and parallel, the following holds: (i) the velocity does not vary along the magnetic field lines; (ii) there is a lowerorder Hartmann layer at the free surface, which is formed to relax traction at the free surface, produced by the core flow.

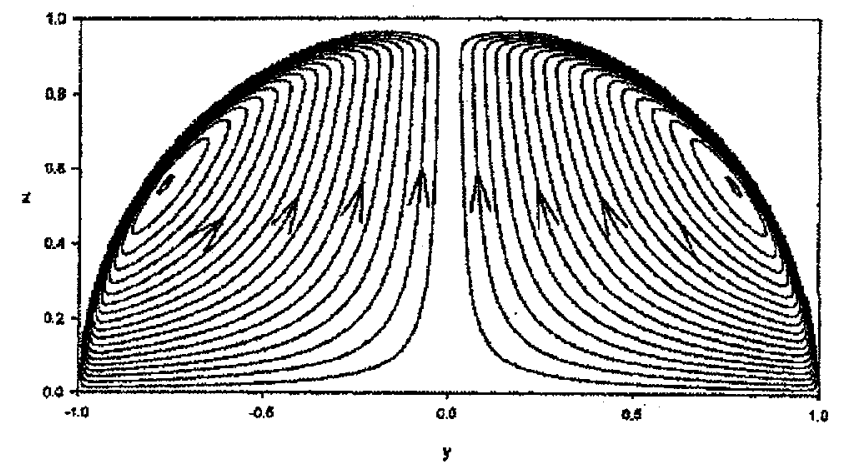

Figure 4. Rivulet in a parallel field: electric current lines for $H a=100$ and contact angle $\theta=\pi / 2$.

\section{Thermocapillary convection in a liquid metal jet}

The second problem to be discussed here is that of a two-dimensional thermocapillary convection (Fig. 5). This problem is related to heat transfer in a jet (problem 4 ), which is of primary concern for the divertor. The heat flux in the divertor region may reach values of $\sim 50$ $\mathrm{MW} / \mathrm{m}^{2}$, most of which is to be absorbed by the jet curtain. This leads to considerable variation of the surface tension, $\beta_{*}$, with temperature, $T_{*}$, and thus to thermocapillary convection. We assume that the surface tension of the liquid metal varies linearly with temperature, i.e.

$\beta_{*}\left(T_{*}\right)=\beta_{*}\left(T_{0}\right)-\left(T_{*}-T_{0}\right) \frac{d \beta_{*}}{d T_{*}}$.

If this effect is sufficiently strong, the resulting thermocapillary flow may lead to a significant deformation of the jet cross-section, and further to the disintegration of the jet. An analogous problem for a liquid metal drop has been discussed in [5].

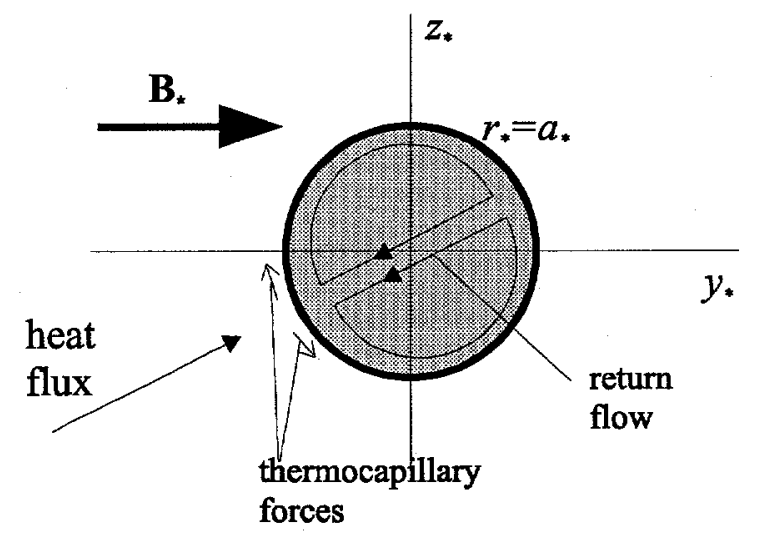

Figure 5. Thermocapillary convection in a jet crosssection: schematic diagram.

Consider now the steady convective motion of a viscous, electrically conducting, incompressible fluid in 
a round, infinite jet (Fig. 5) in a strong, uniform magnetic field $\mathbf{B}_{*}=B_{0} \hat{\mathbf{y}}$. The jet flows in the $x_{*}$ direction. Cylindrical co-ordinates $\left(r, \theta, x_{*}\right)$ will be used as well. The relation between the two systems is $y_{*}=r_{*} \cos \theta, z_{*}=r_{*} \sin \theta$. The jet is surrounded by vacuum. The radius of the jet cross-section is $a_{*}$. At this stage the problem is supposed to be independent of $x_{*}$, so that it is two-dimensional, in the $\left(y_{*}, z_{*}\right)$-plane.

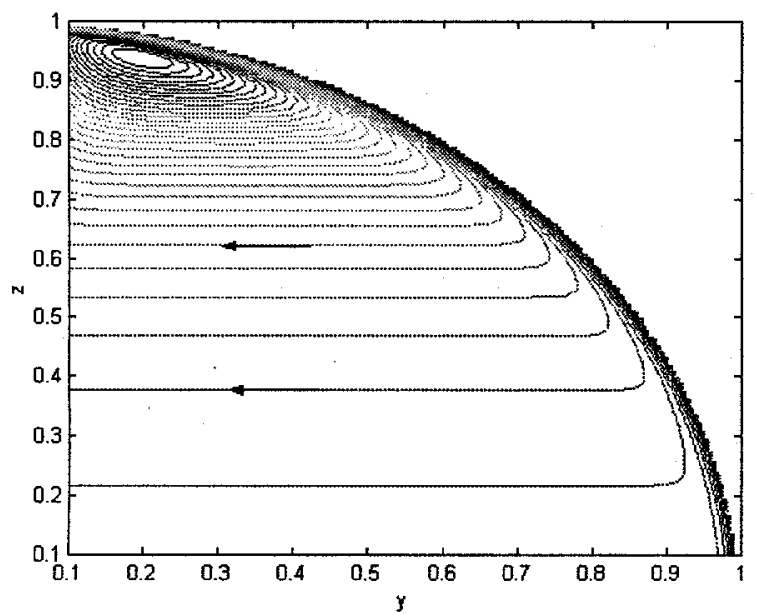

Figure 6 . Thermocapillary convection in a jet: streamlines for $z>0.1, y>0.1$, and for $T=-y, H a=$ 100 .

Variation of the surface tension with temperature, represents the driving force, and creates a traction at the free surface. In dimensionless form this gives:

$$
\frac{\partial v_{r}}{\partial \theta}+\frac{\partial v_{\theta}}{\partial r}-\frac{v_{\theta}}{r}=\frac{\partial T}{\partial \theta}, \quad \text { at } r=1 .
$$

Typical results are shown in Fig. 6 . At the jet surface a Hartmann layer is formed, where the flow is dragged from the hot to cold region by thermocapillary forces. The flow returns via the core. More details are given in [6]. Deformation of the jet owing to this effect is currently being investigated.

\section{References}

1. S. Molokov and C.B. Reed, 1999 "Review of freesurface MHD experiments and modelling", $A N L$ Report No. ANL/TD/TM99-08.

2. S. Oshima, R. Yamane, Y. Mochimaru and $\mathrm{K}$. Sudo, Bull. JSME., 253, 2078-2085 (1986).

3. V.V. Baranov et al. Magnetohydrodynamics, 30, 460-467 (1994).

4. S. Molokov and C.B. Reed, 2000 "Fully developed magnetohydrodynamic flow in a rivulet" $A N L$ Report No. ANL/TD/TMO0-12.

5 .A. Gailitis, and G. Gerbeth, 1987, Magnetohydrodynamics, 27, 166-169.

6. I. Cox, 1999 MSc Thesis, Coventry University 